\title{
Chemoresistance to paclitaxel induces epithelial- mesenchymal transition and enhances metastatic potential for epithelial ovarian carcinoma cells
}

\author{
HIROAKI KAJIYAMA, KIYOSUMI SHIBATA, MIKIO TERAUCHI, MAMORU YAMASHITA, \\ KAZUHIKO INO, AKIHIRO NAWA and FUMITAKA KIKKAWA
}

\author{
Department of Obstetrics and Gynecology, Nagoya University Graduate School of Medicine, \\ Tsuruma-cho 65, Showa-ku, Nagoya 466-8550, Japan
}

Received March 22, 2007; Accepted May 9, 2007

\begin{abstract}
The aim of this study was to assess paclitaxel resistant-epithelial ovarian carcinoma (EOC) cells for cellular morphology, motility, and molecular changes consistent with epithelial-mesenchymal transition (EMT). The human EOC cell lines NOS-2, TAOV and SKOV-3 were continuously exposed to increasing doses of paclitaxel to establish three stable cell lines resistant to paclitaxel (NOS-PR, TAOV-PR, and SKOV-PR cells, respectively). Using these cell lines, cellular functions such as motility, invasive ability, and proliferative potential were assessed. Several molecules involved in EMT or cell invasiveness were assessed using Western blot analysis. In a peritoneal metastasis model using mice inoculated with NOS-2 or NOS-PR cells, we investigated the differences of peritoneal dissemination and survival time. NOS2-PR cells showed phenotypic changes consistent with EMT; with spindle-shaped morphology and enhanced pseudopodia formation. Western blot analysis revealed decreased expression of the epithelial adhesion molecule, E-cadherin and an increase in mesenchymal markers such as vimentin, fibronectin and smooth-muscle actin in NOS-PR cells compared to NOS-2 cells. The NOS2-PR cells displayed increased expression of Snail and Twist, EMT-regulatory transcription factors. Migratory potential in a wound assay and metastatic potential to the peritoneum of mice were markedly enhanced in NOS2-PR cells compared to NOS-2 cells. These data suggest that there is a possible link between chronic paclitaxelresistance and induction of the EMT in EOC cells. It is possible that therapeutic benefits such as the restoration of chemosensitivity or suppression of metastasis will be enabled by gaining further insight into the mechanisms underlying chemoresistance and EMT.
\end{abstract}

Correspondence to: Dr Hiroaki Kajiyama, Department of Obstetrics and Gynecology, Nagoya University Graduate School of Medicine, Tsuruma-cho 65, Showa-ku, Nagoya 466-8550, Japan

E-mail:kajiyama@med.nagoya-u.ac.jp

Key words: paclitaxel, chemoresistance, epithelial-mesenchymal transition, migration, epithelial ovarian carcinoma, peritoneal metastasis

\section{Introduction}

Epithelial ovarian carcinoma (EOC) is a major cause of death among the gynecological malignancies (1). EOC has a poor prognosis, primarily due to its late symptomatology, and is often associated with multiple intraperitoneal disseminations, and frequently with distant metastases. Treatment for advanced EOC is difficult because of both the inability to completely resect diffuse tumor involvement on the peritoneal surface and the eventual resistance of the tumor cells to chemotherapy. Paclitaxel is a first-line chemotherapeutic agent that is effective for the treatment of EOC. Paclitaxel exerts its effect through stabilization of microtubules, induction of cell cycle arrest in $\mathrm{G} 2-\mathrm{M}$, and activation of proapoptotic signaling $(2,3)$. However, in spite of the comparatively high sensitivity of EOC to paclitaxel, the prognosis of advanced or recurrent cases remains poor since most deaths are the result of metastasis that is refractory to conventional chemotherapy. To overcome the paclitaxel-resistance, a variety of additional molecular-targeting therapies combined with paclitaxel have been investigated $(4,5)$.

Tumor cells progress from a non-invasive to an invasive and malignant phenotype through a series of metastatic steps $(6,7)$. These steps involve a change in morphology of the epithelioid or cobblestone appearance to a fibroblastic form through a process referred to as the epithelial-mesenchymal transition (EMT) (8-10). EMT is a process initially observed during embryonic development in which cells lose epithelial characteristics such as downregulation of E-cadherin and gain mesenchymal properties that increase motility and invasion (11). According to several reports, the EMT is induced by several growth factors involved in tumor-progression processes such as VEGF, TGF- $\beta$, or EGF $(12,13)$. Furthermore, with respect to chemoresistance, recent studies demonstrated that there is a close link between EMT and insensitivity to several growth factors or chemotherapeutic agents $(12,14,15)$. Hiscox et al demonstrated that Tamoxifen-resistant MCF7 cells showed loss of association between $\beta$-catenin and E-cadherin, and additionally showed increased cytoplasmic and nuclear $\beta$-catenin and elevated transcription of $\beta$-catenin target genes known to be involved in tumor progression and EMT (15).

Clinically, EOC frequently shows sudden and multiple metastasis following continuous treatment with chemotherapeutic agents, including paclitaxel. Based on this 
observation, in the current study, we examined the EMT-like change in paclitaxel-resistant EOC cells, which display enhanced motile and invasive behavior in vitro and increased formation of peritoneal metastasis in a mouse model compared to their paclitaxel-sensitive counterparts. Our data demonstrate that the development of paclitaxel-resistance in EOC cells is accompanied by a transition from an epithelial to a mesenchymal phenotype.

\section{Materials and methods}

Cell culture. We used three human EOC cell lines (SKOV-3, NOS-2, and TAOV) in this study. SKOV-3, NOS-2, and TAOV cells were cultured and maintained as described previously $(6,16)$. The cell lines were maintained in RPMI1640 supplemented with $10 \%$ fetal calf serum (FCS) and penicillin-streptomycin at $37^{\circ} \mathrm{C}$ in a humidified atmosphere of $5 \% \mathrm{CO}_{2}$.

Establishment of paclitaxel-resistant EOC cells. The above cells were washed thoroughly with PBS, and transferred to RPMI-1640 medium containing 10\% FCS and penicillinstreptomycin. The cells were continuously exposed to paclitaxel for more than 6 months, during which time the medium was replaced every 3 days and the cell cultures were passaged by trypsinization after $70 \%$ confluency was reached. Gradually, these cells displayed resistance to the growthinhibitory properties of paclitaxel. These cell lines, designated paclitaxel-resistant, were cultured for a further 3 months in medium containing paclitaxel before characterization studies. Through this process, we generated three paclitaxel-resistant EOC cell lines (NOS-PR, TAOV-PR, and SKOV-PR cells).

Wound assay. Cells were grown in 10-cm culture dishes. When the cells became confluent, the medium was replaced with fresh RPMI-1640 containing 1\% FCS. Confluent cells were wounded with a uniform scratch using sterile pipette tips, rinsed to remove debris, then incubated in culture medium containing $1 \%$ FCS for $18 \mathrm{~h}$. Quantitative analysis of the wound healing index expressed as percentage was calculated using 20 randomly chosen distances across the wound at $0 \mathrm{~h}$ and $18 \mathrm{~h}$, divided by the distance measured at $0 \mathrm{~h}$.

In vitro invasion assay. Cell invasiveness was evaluated using 24-well Matrigel invasion chambers (Becton Dickinson Labware). Cells were suspended in the upper chamber at a final concentration of $10 \times 10^{4} / \mathrm{ml}$ in $200 \mu \mathrm{l}$ of RPMI-1640 supplemented with $1 \%$ FCS. The lower chamber contained $700 \mu \mathrm{l}$ of RPMI-1640 supplemented with $1 \%$ FCS. After $18 \mathrm{~h}$ of incubation, the tumor cells remaining on the upper surface of the filters were removed by wiping with cotton swabs, and the invading cells on the lower surface were stained with May-Grünwald Giemsa staining. The number of cells on the lower surface of the filters was counted under a microscope at a magnification of $\mathrm{x} 200$. The invasion assay was performed in triplicate in four individual experiments.

In vitro cell proliferation assay. Cells were plated in triplicate at a density of 1500 cells in a $200-\mu 1$ volume in 96-well plates, and cultured for 1 to 4 days. Cell viability was assayed using a modified tetrazolium salt MTT assay performed using a CellTiter 96 Aqueous One Solution cell proliferation assay kit (Promega) according to the manufacturer's instructions. Absorbance was measured at $492 \mathrm{~nm}$ using a microplate reader (Labsystems, Multiskan Bichromatic).

Paclitaxel chemosensitivity assay. Cells were plated in triplicate in 96-well plates at a density of 5000 cells in a volume of $200 \mu 1$ of culture medium containing $10 \%$ FCS. After incubation for $24 \mathrm{~h}$ at $37^{\circ} \mathrm{C}$, the medium was replaced by fresh medium with or without various concentrations of paclitaxel (Bristol Myers Squib, Tokyo, Japan). After an additional $72 \mathrm{~h}$, cell viability was assayed using a modified MTT assay as described above. IC50 values were defined as the concentrations resulting in a 50\% reduction in growth as compared with control cell growth. Experiments were performed in triplicate.

Western blot analysis. Western blot analysis was performed as described previously (16). Briefly, $30 \mu \mathrm{g}$ of cell lysates were electrophoresed in a sodium dodecyl sulfate polyacrylamide gel under reducing conditions. After electrophoresis, the proteins were transferred electrophoretically to an Immobilon membrane (Millipore, Bedford, MA). After blocking, the membrane was incubated for $1 \mathrm{~h}$ with the respective anti-human primary antibody at the recommended dilution [anti-E-cadherin, fibronectin, vimentin, matrix metalloproteinase-2 (MMP-2), tissue inhibitor of metalloproteinase-II (TIMP-II), Snail, Twist, $ß$-actin (Santa Cruz), smooth muscle actin (SMA) (Progen), membrane-type I-MMP (MT-MMP) (Chemicon), TIMP-I (Oncogene)]. The membrane was washed 3 times with Tween/ PBS for 15 min each time, and then incubated with the appropriate secondary antibody for $1 \mathrm{~h}$. After washing with Tween/PBS, the membrane was treated with ECL-Western blotting detecting reagent (Amersham Biosciences K.K., Tokyo, Japan).

Immunofluorescence staining. NOS-2 and NOS-PR cells were grown in chamber slides (Nalge Nunc International). They were fixed for 15 min with $4 \%$ paraformaldehyde and washed several times with PBS. Coverslips were incubated in blocking solution containing 2\% BSA in PBS for $1 \mathrm{~h}$, and incubated with the appropriate primary antibodies [anti-mouse E-cadherin and anti-rabbit ß-catenin antibodies (Santa Cruz Biotech, CA)] for $1 \mathrm{~h}$ at room temperature. After incubation with appropriate secondary antibodies [E-cadherin, goat antimouse IgG-FITC; $\beta$-catenin, goat anti-rabbit rhodamineconjugated IgG (Santa Cruz Biotech)], fluorescence was visualized by epifluorescence confocal microscopy (Pascal, BioRad).

In vivo studies. Female nude mice (BALB/c) at 5 weeks of age were obtained from Japan SLC (Nagoya, Japan). The treatment protocol followed the guidelines for animal experimentation adopted by Nagoya University. NOS2 and NOS2-PR cells $\left(1 \times 10^{7}\right.$ cells $/ 0.5 \mathrm{ml}$ of medium/mouse) were injected into mice intraperitoneally to generate peritoneal metastasis of EOC in the mouse model. To examine the metastatic potential of the cells, mice were sacrificed 25 days after the injection of carcinoma cells, and the production of ascites and formation 
A

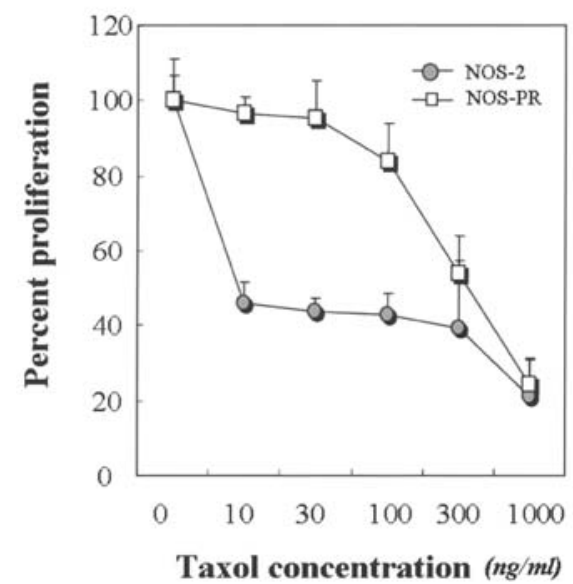

B
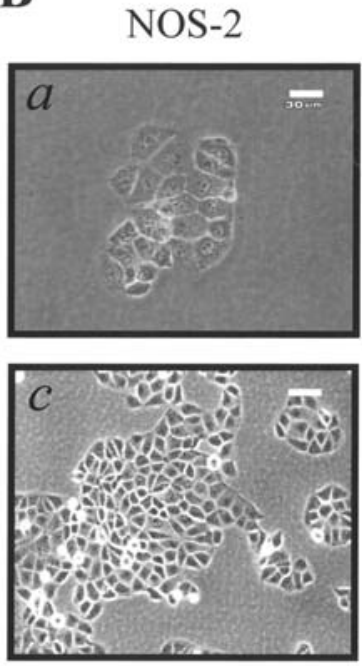

NOS-PR
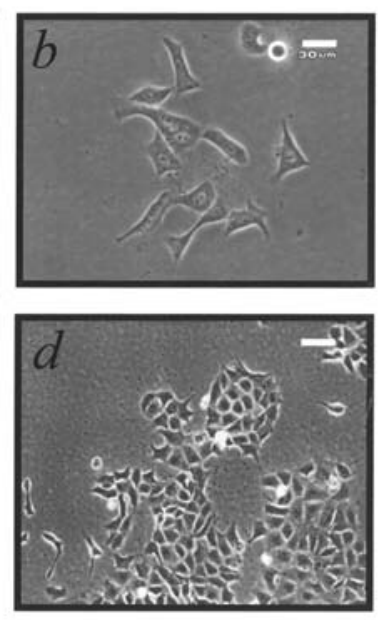

Figure 1. (A) Paclitaxel-sensitivity assay in parental NOS-2 and resistant NOS-PR cells. IC50 values of NOS-2 and NOS-PR cells were 9.3 ng/ml and $367.3 \mathrm{ng} / \mathrm{ml}$, respectively. (B) Parental NOS-2 cells displayed an epithelioid and cobblestone appearance. Cell morphology was observed by microscopy at x200 magnification ( $a$ and b) and x100 magnification (c and d). In parental NOS-2 cells, the shape of marginal cells was rounded, showing little formation of pseudopodia (b and d). In contrast, the phenotypic changes observed in NOS-PR cells included loss of cell polarity causing a spindle-shaped morphology, and increased formation of pseudopodia ( $\mathrm{a}$ and c). Bars, $30 \mu \mathrm{m}$ ( $\mathrm{a}$ and b) and $60 \mu \mathrm{m}$ (c and d).

of intraperitoneal dissemination were evaluated $(n=4)$. In addition, survival time was compared between the two groups $(n=7)$.

Statistical analysis. For data of in vitro experiments, statistical comparisons among groups were performed using the nonpaired Student's t-test. Differences between groups were considered statistically significant at $\mathrm{P}<0.05$. Data are expressed as mean \pm SD. Moreover, the Kaplan-Meier method was used to generate survival curves, and comparisons were performed using log-rank tests. $\mathrm{P}<0.05$ was considered significant.

\section{Results}

Acquisition of paclitaxel resistance induces morphologic changes consistent with EMT in EOC cells. We first confirmed the resistance to paclitaxel of NOS-PR cells. Fig. 1A shows the paclitaxel-sensitivity assay of parental NOS-2 and resistant NOS-PR cells. Chemoresistance to paclitaxel was found in NOS-PR cells compared to NOS-2 cells. The IC50 values of NOS-2 and NOS-PR cells were $9.3 \mathrm{ng} / \mathrm{ml}$ and $367.3 \mathrm{ng} / \mathrm{ml}$, respectively. The IC50 value of NOS-PR cells was considered to correspond to clinical paclitaxel-resistance. The parental NOS-2 cells displayed an epithelioid and cobblestone appearance. The shape of marginal cells was rounded, showing little formation of pseudopodia (panel a and c). In contrast, the phenotypic changes observed in NOS-PR cells included loss of cell polarity, causing a spindle-shaped morphology, and increased formation of pseudopodia (panel $b$ and $d$ ). According to these alternations, NOS-PR cells were thought to have acquired a mesenchymal phenotype.

Enhanced migratory and invasive potential of NOS-PR cells. We next compared various cellular functions between the NOS-PR and parental NOS-2 lines, such as migratory ability, invasiveness, and proliferative potential. Wound assays were performed to compare the migratory potential of NOS-PR and parental NOS- 2 cells. At $18 \mathrm{~h}$, the NOS-PR cells showed a 2.7-fold increase in the number of cells migrating across the wound $(\mathrm{P}<0.001$; Fig. 2A and B). Increased formation of pseudopodia was observed in the edge of the wounds of NOS-PR cells compared to those of NOS-2 cells (data not shown). In addition, we compared the invasive potential between NOS-PR cells and NOS-2 cells using a Matrigel Boyden chamber. The capacity of NOS-PR cells to invade through a Matrigel-coated membrane was 2.4-fold greater compared with that of the parental NOS-2 cells (Fig. 2C, $\mathrm{P}<0.005)$.

We further investigated the proliferative potential of NOS-2 and NOS-PR cells at various times after plating by assessing cell viability using a modified MTT assay. At 72 and 96 h, NOS-PR cell lines exhibited a slight (not significant) decrease in cell growth compared with the parental NOS-2 cells (Fig. 2D).

The expression of EMT- and cell invasiveness-related molecules in NOS-2 and NOS-PR cells. To determine whether the acquisition of paclitaxel resistance induced specific molecular changes consistent with EMT, Western blotting was performed on cell lysates from the NOS-PR and parental NOS-2 cells (Fig. 3A). Expression of the epithelial adhesion molecule, E-cadherin was decreased in NOS-PR cells compared with NOS-2 cells. The expression of mesenchymal markers such as vimentin, SMA, and fibronectin was increased in NOS-PR cells (Fig. 3A). Moreover, the expression of several proteolytic proteins involved in cellular invasiveness, including MMP-2 and MT1-MMP was concurrently increased in NOSPR cells. In contrast, the expression of TIMP-1 and TIMP-2 was downregulated in NOS-PR cells (Fig. 3B). Furthermore, the expression of EMT-related transcription factors Snail and Twist was increased in NOS-PR cells compared with NOS-2 cells (Fig. 3C). To further investigate the possibility that 
A

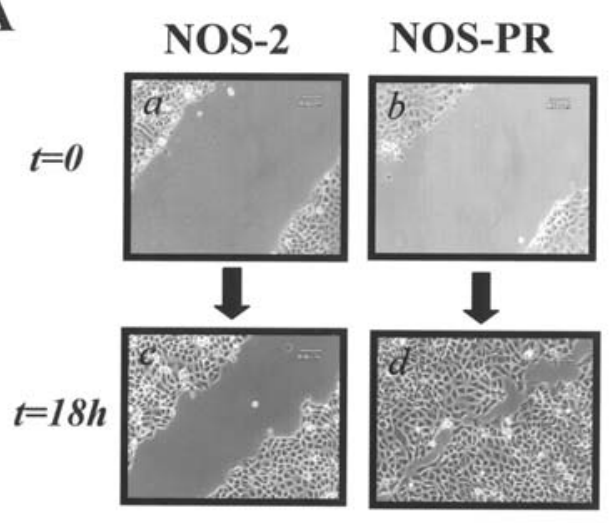

B

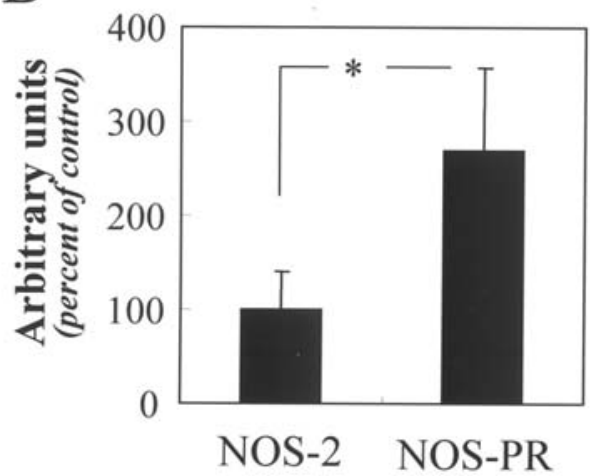

C

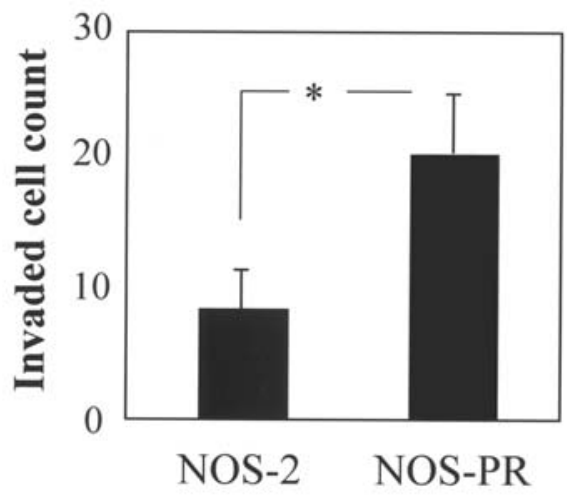

D

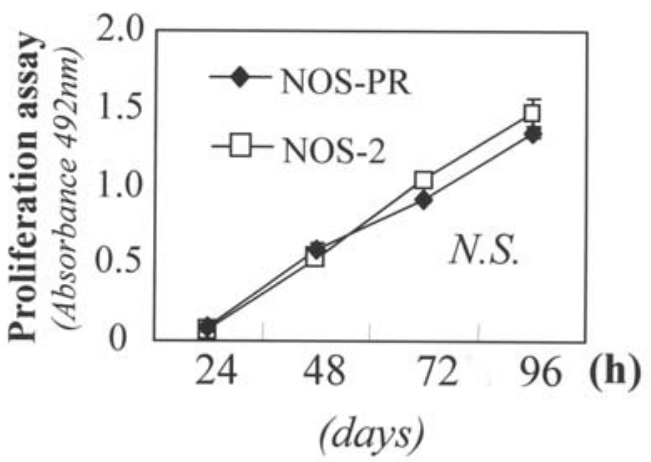

Figure 2. (A) Wound assays were performed to compare the migratory potential of NOS-PR and parental NOS-2 cells. Panels a and b; NOS-2 and NOS-PR cells at time zero, respectively. Panels c and d; NOS-2 and NOS-PR cells after $18 \mathrm{~h}$ of incubation. (B) Quantitative results are shown as wound healing index, which was calculated using 20 randomly chosen distances across the wound at 0 and $18 \mathrm{~h}$. Y-axis indicates percent of control (the migratory potential of NOS-2 cells). (C) The invasive potentials of NOS-PR cells and NOS-2 cells assessed using Matrigel Boyden chambers. The capacity of NOS-PR cells to invade through a Matrigel-coated membrane was 2.4-fold greater than that of NOS-2 cells ( $\left.{ }^{*}<0.005\right)$. (D) Proliferative potentials of NOS-2 and NOS-PR cells at various times after plating assessed as cell viability using a modified MTT assay. At 72 and 96 h, NOS-PR cells exhibited a slight decrease in cell growth compared with the parental NOS-2 cells (not significant).

A

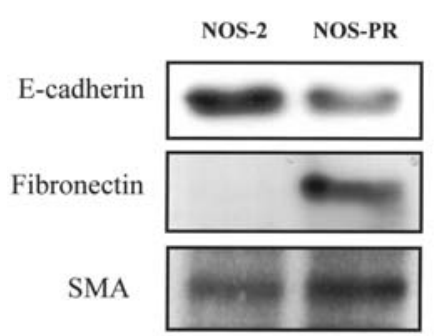

Vimentin

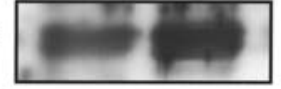

$\beta$-actin

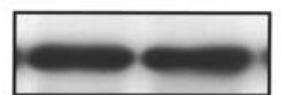

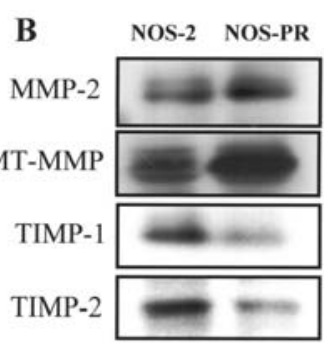

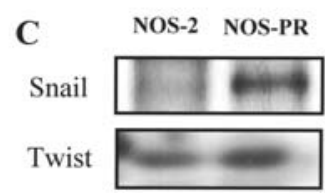

Figure 3. Western blot analysis. (A) The differences of epithelial and mesenchymal markers in NOS-2 compared to NOS-PR cells. Expression of E-cadherin was decreased in the NOS-PR cells compared with the NOS-2 cells. Concurrently, marked increases of the expression of mesenchymal markers such as vimentin, SMA, and fibronectin were observed. (B) The expression of MMPs and TIMPs in NOS-2 and NOS-PR cells. The expression of MMP-2 and MT1-MMP was decreased in NOS-PR cells. In contrast, the expression of TIMP-1 and TIMP-2 was upregulated in NOS-PR cells. (C) Increased expression of the EMT-related transcription factors Snail and Twist was observed in NOS-PR cells compared with NOS-2 cells.

paclitaxel-resistance leads to changes in the cellular localization of E-cadherin and B-catenin, immunofluorescence staining was performed on NOS-PR and NOS-2 cells. As shown in Fig. 4, E-cadherin and $\beta$-catenin were localized and organized in the membrane in NOS-2 cells. In contrast, in NOS-PR cells, the cell-shape was disorganized and E-cadherin and B-catenin seemed to be dispersed throughout the cytoplasm.

The occurrence of EMT and increased expression of EMTrelated molecules in PAC-resistant TAOV and SKOV-3 cells. We further examined whether the occurrence of EMT and enhanced expression of EMT-related proteins were observed in other EOC cell lines resistant to paclitaxel (TAOV-PR, SKOV-PR cells). The IC50 values of TAOV, TAOV-PR, SKOV-3, and SKOV-PR cells were 6.7, 452.2, 50.7, and 532.1, respectively. As shown in Fig. 5A, the parental TAOV cells showed an epithelioid, cobblestone appearance, like NOS-2 cells. On the other hand, the morphology of TAOV-PR cells was mixed type, showing an epithelioid and a short-bipolar/ fibroblastic pattern with formation of pseudopodia. In addition, a remarkable morphological change was observed in SKOV-PR cells. While the parental SKOV-3 cells showed a short spindle-shaped morphology, SKOV-PR cells had a long spindle/fibroblastic morphology with a scattered and 
A

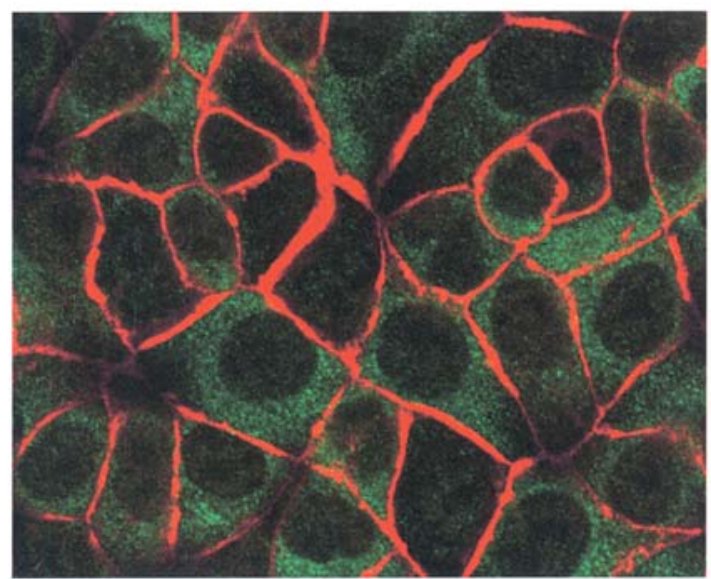

B

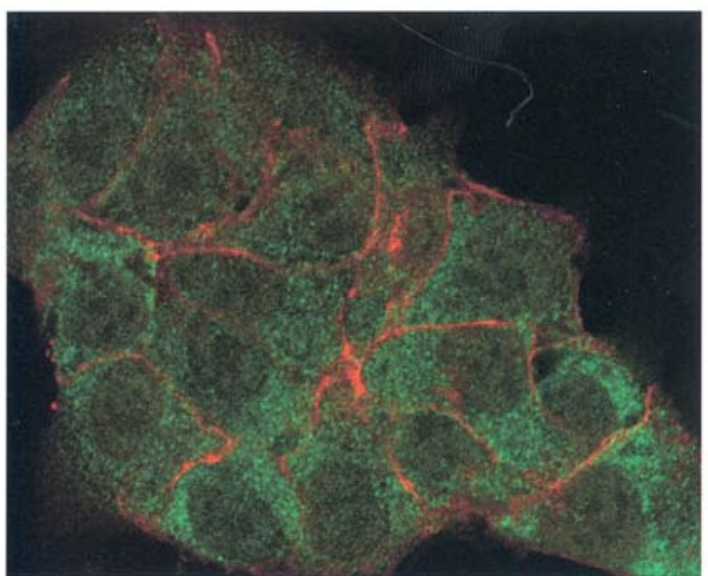

Figure 4. The expression and localization of E-cadherin (green) and ß-catenin (red) in NOS-2 and NOS-PR cells assessed using immunofluorescence staining. E-cadherin and B-catenin were localized and organized in the membrane in NOS-2 cells. In contrast, in NOS-PR, they seemed to be dispersed throughout the cytoplasm (arrowheads).

A
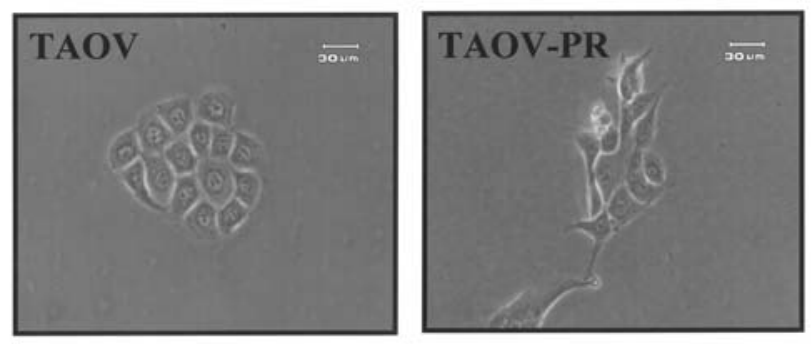

B

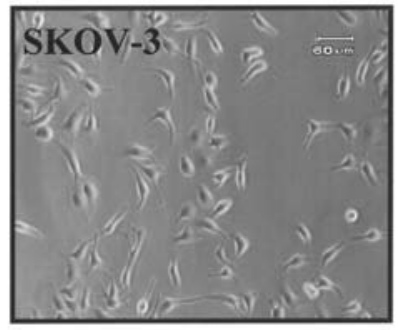

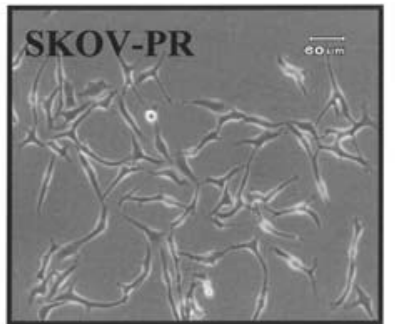

C

TAOV TAOV-PR

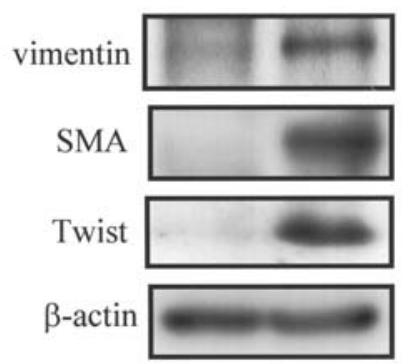

D

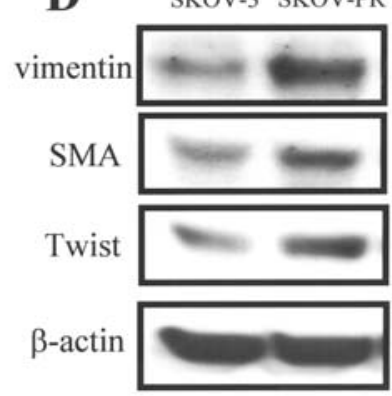

Figure 5. The occurrence of EMT and increased expression of EMT-related molecules in PAC-resistant TAOV and SKOV-3 cells. (A) Cell morphology of TAOV and TAOV-PR cells was observed by microscopy at x200 magnification. Bars, $30 \mu \mathrm{m}$. Parental TAOV cells showed an epithelioid, cobblestone appearance, like NOS-2 cells. On the other hand, the morphology of TAOV-PR cells was mixed type, showing an epithelioid and a short-bipolar/fibroblastic pattern with formation of pseudopodia. (B) Cell morphology of SKOV-3 and SKOV-PR cells was observed by microscopy at x100 magnification. Bars, $60 \mu \mathrm{m}$. A remarkable morphological change was observed in SKOV-PR cells. While parental SKOV-3 cells had a short spindle-shaped morphology, SKOV-PR cells had long spindle/fibroblastic morphology with a scattered and unorganized growth pattern. (C and D) The expression of mesenchymal markers as shown by Western blot analysis. Enhanced expression of vimentin, SMA, and Twist was observed in both TAOV-PR (C) and SKOV-PR cells (D) compared to the parental TAOV and SKOV-3 cells.

unorganized growth pattern (Fig. 5B). Furthermore, increased levels of mesenchymal markers such as vimentin, SMA, and Twist were observed in both TAOV-PR and SKOV-PR cells compared to the respective parental cells (Fig. 5C andD).

Enhanced metastatic potential of NOS-PR cells to the murine peritoneum. Finally we investigated the ability to metastasize to the peritoneal cavity using a mouse intraperitoneal model. Carcinomatous peritonitis with a large amount of bloody ascites was observed approximately 25 days after the inoculation of NOS-PR cells into mice (Fig. 6A). Fig. 6B shows the intraabdominal appearance of the mice 25 days after the inoculation of NOS-PR and NOS-2 cells. In the former, a number of disseminated tumors were observed throughout the whole 
A

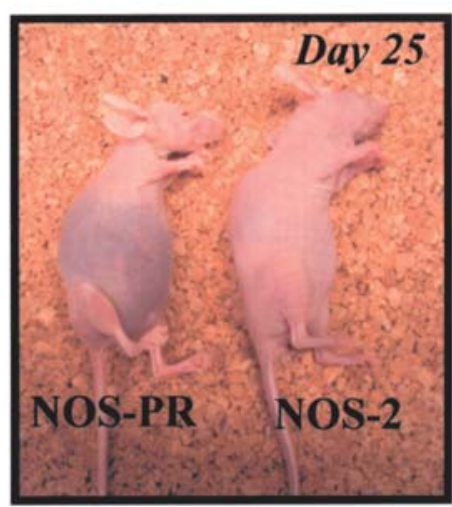

C

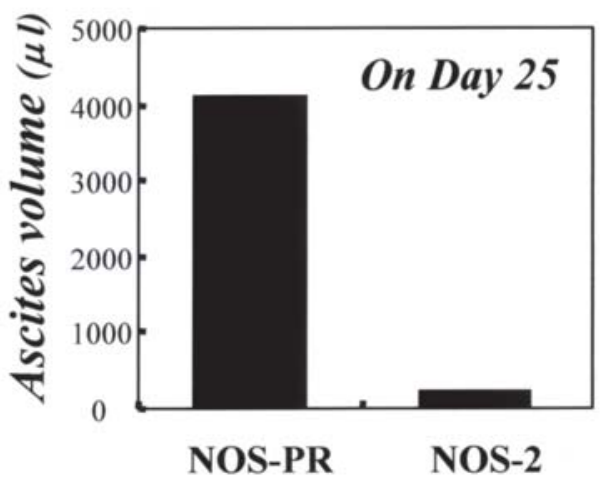

B

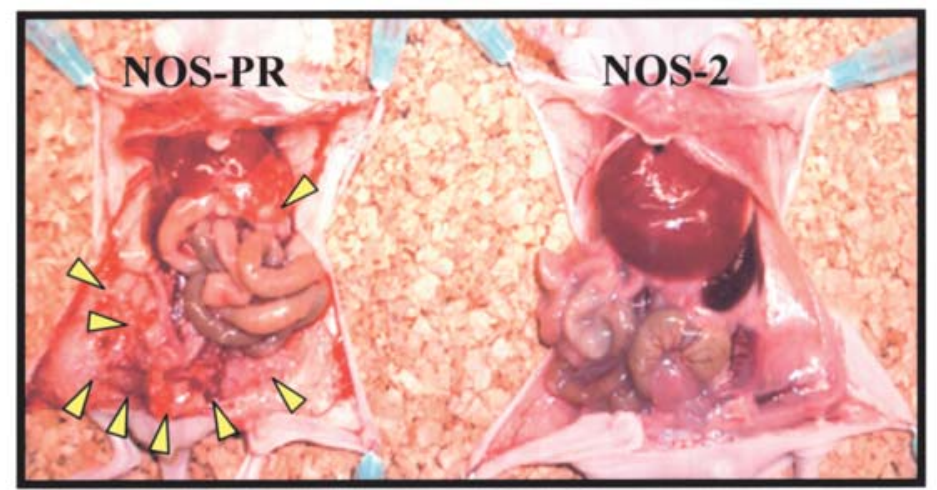

D

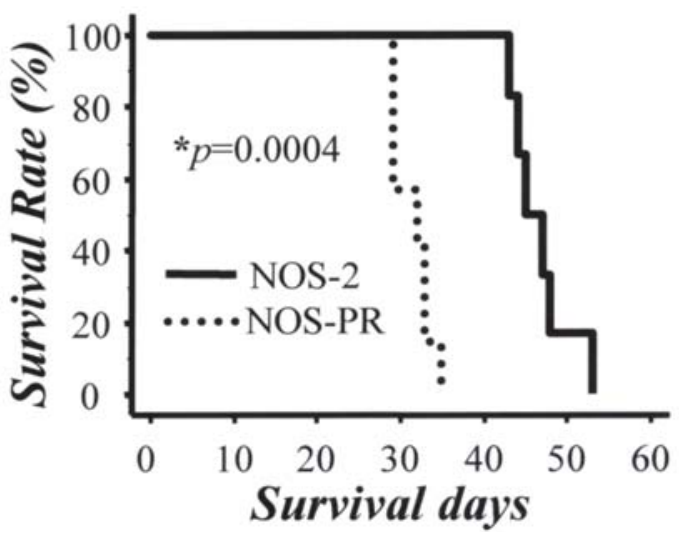

Figure 6. NOS2 and NOS2-PR cells $\left(1 \times 10^{7}\right.$ cells $/ 0.5 \mathrm{ml}$ of medium/mouse) were injected intraperitoneally to generate peritoneal metastasis of EOC in the mouse model. To examine their peritoneal metastatic potential, mice were sacrificed 25 days after the injection of carcinoma cells ( $\mathrm{n}=4$ ). (A) The general appearance of the mice 25 days after inoculation of NOS-PR and NOS-2 cells. Carcinomatous peritonitis with a large amount of bloody ascites was observed approximately 25 days after the inoculation of NOS-PR cells into mice. (B) The intraabdominal appearance of the mice 25 days after inoculation of NOS-PR and NOS-2 cells. Arrowheads show disseminated tumors throughout the murine peritoneal cavity. (C) Amount of ascites at the same time of autopsy ( $\mathrm{n}=4$ ). Mice injected with NOS-PR cells had a markedly larger amount of ascites than mice injected with NOS-2 cells. (D) Survival time was compared between the two groups (each group consisted of seven mice). The Kaplan-Meier method was used to generate survival curves, and comparisons were performed using log-rank tests. The mice injected with NOS-PR cells survived for a significantly shorter period than those injected with NOS-2 cells [mean survival, $46.7 \pm 2.43$ days (NOS-2 cells) and 31.4 \pm 3.61 days (NOS-PR cells), respectively, $\mathrm{P}=0.0004]$.

peritoneal cavity. In contrast, a mouse injected with NOS-2 cells had no macroscopically visible disseminated tumor, and had a significantly smaller amount of ascites at the same time of autopsy (Fig. 6C). Fig. 6D shows the survival curves of these two groups. All mice finally died of carcinomatous peritonitis. However, the mice injected with NOS-PR cells had significantly shorter survival than those injected with NOS-2 cells [mean survival, 46.7 \pm 2.43 days (NOS-2) and $31.4 \pm 3.61$ days $(\mathrm{NOS}-\mathrm{PR}), \mathrm{p}=0.0004]$.

\section{Discussion}

Metastatic processes require reduction of E-cadherin expression to disrupt the adherence junction complex and the upregulation of proteolytic enzymes degrading extracellular matrix complexes, resulting in the facilitation of invasion into the surrounding tissues. Thus, many studies have focused on the relationship between loss of E-cadherin expression and the invasive and metastatic process. The prognosis of patients with EOC is most likely related to the degree of peritoneal dissemination $(8,10,11)$. Numerous immunohistochemical studies have demonstrated that loss of E-cadherin expression is frequently associated with a poorer survival rate in patients with EOC (17-19). Besides the enhanced metastatic ability induced by loss of E-cadherin, chemoresistance is the major cause of the poor prognosis of EOC patients. Cisplatin and paclitaxel are major chemotherapeutic agents whose use results in improved outcomes; however, five-year survival is only $20 \%$ in advanced EOC patients in spite of the fact that most tumors are chemosensitive during the initial treatment (20). Clinically, enhanced metastatic ability and chemoresistance are frequently concurrent during the therapeutic course of EOC and seem to be linked to each other in light of the evolution of tumors toward increasingly malignant characteristics. However, the molecular mechanisms associated with these changes have not been elucidated.

In our current analysis, we demonstrated that EOC cells acquired an increased capacity for in vitro migration and invasion during the development of resistance to paclitaxel. In addition, we demonstrated that paclitaxel-resistant EOC 
cells lost their epithelial features and acquired mesenchymal characteristics, which are events characteristic of the EMT. Furthermore, in our animal model, the mice injected with NOS-PR cells had significantly shorter survival and enhanced peritoneal dissemination compared to those injected with NOS- 2 cells. According to this result, tumors that have acquired paclitaxel-resistance may have characteristics that favor easily spreading into the peritoneal cavity, resulting in an increased chance of adhering to the mesothelium and enhanced formation of microscopic or macroscopic peritoneal metastasis. Our current evidence seems to be associated with the clinical observation that sudden peritoneal recurrence or another type of lymphovascular metastasis occurs during continuous treatment with chemotherapeutic agents, including paclitaxel. Our data indicate a possible link between paclitaxel-resistance and facilitated metastatic potential of EOC.

We obtained molecular evidence that the EMT-like changes in the NOS-PR cell line were associated with an increase in the transcription factors Snail and Twist. Snail and Twist are major transcription factors responsible for the development of the EMT. They can bind directly to the E-boxes on the E-cadherin promoter to repress its transcription (21). Clinically, in our preliminary study in 82 EOC patients, Twist was not associated with any of the clinicopathological parameters examined. However, positive Twist expression significantly predicted poorer overall survival and progressionfree survival when compared with negative expression. Wang et al demonstrated that increased Twist was responsible for the development of acquired paclitaxel-resistance in nasopharyngeal carcinoma cells and ectopic expression of Twist conferred resistance to microtubule-disrupting agents, including paclitaxel (22). Conversely, our present study revealed that paclitaxel-resistance led to upregulation of Twist expression and subsequent occurrence of EMT. Although the detailed mechanism is still under investigation, it is possible that the increased expression of Twist or Snail is involved in the mechanism of the occurrence of EMT in paclitaxel-resistant cells. Chemotherapeutic agents, including paclitaxel, generally induce tumor regression through apoptosis, which is modulated through a series of proto-oncogenes and tumor suppressor genes. However, alterations in the regulation of such apoptotic processes potentially may lead to increased expression of such EMT-inducible factors and result in the failure of therapy. Although the detailed mechanisms underlying paclitaxelresistance and EMT are still under investigation, therapeutic strategies targeting these EMT-promoting molecules may restore the chemosensitivity of EOC cells to paclitaxel.

As far as we know, this is the first report showing that chronic paclitaxel-resistance may lead to the EMT and a subsequent increase of the potential to metastasize to the peritoneal cavity. An earlier study by Yang et al showed that oxaliplatin-resistant colorectal cells displayed EMT-like changes with decreased expression of E-cadherin and an increase in the mesenchymal markers vimentin and Snail (14). This evidence is consistent with our current results.

In conclusion, we have shown here that the development of paclitaxel-resistance in EOC cells is accompanied by inducible EMT-like changes with increased potential to metastasize to the peritoneal cavity of nude mice. Further elucidation of the relationship between paclitaxel-resistance and EMT should make it possible to develop new therapeutic approaches for the treatment of chemo-refractory tumors in the future.

\section{References}

1. Brun JL, Fevler A, Chene G, Saurel J, Brun G and Hocke C: Long-term results and prognostic factors in patients with epithelial ovarian cancer. Gynecol Oncol 78: 21-27, 2000.

2. Horwitz SB: Taxol (paclitaxel): mechanisms of action. Ann Oncol 5 (suppl 6): S3-S6, 1994.

3. Wang TH, Wang HS and Soong YK: Paclitaxel-induced cell death: where the cell cycle and apoptosis come together. Cancer 88: 2619-2628, 2000.

4. Taxman DJ, MacKeigan JP, Clements C, Bergstralh DT and Ting JP: Transcriptional profiling of targets for combination therapy of lung carcinoma with paclitaxel and mitogen-activated protein/extracellular signal-regulated kinase kinase inhibitor. Cancer Res 63: 5095-5104, 2003.

5. Lee JT Jr, Steelman LS and McCubrey JA: Phosphatidylinositol 3'-kinase activation leads to multidrug resistance protein-1 expression and subsequent chemoresistance in advanced prostate cancer cells. Cancer Res 64: 8397-8404, 2004.

6. Kajiyama H, Shibata K, Kikkawa F, et al: Neutral endopeptidase 24.11/CD10 suppresses progressive potential in ovarian carcinoma in vitro and in vivo. Clin Cancer Res 11: 1798-1808, 2005.

7. Kajiyama H, Kikkawa F, Suzuki T, Shibata K, Ino K and Mizutani S: Prolonged survival and decreased invasive activity attributable to dipeptidyl peptidase IV overexpression in ovarian carcinoma. Cancer Res 62: 2753-2757, 2002.

8. Vernon AE and LaBonne C: Tumor metastasis: a new twist on epithelial-mesenchymal transitions. Curr Biol 14: R719-R721, 2004.

9. Savagner P: Leaving the neighborhood: molecular mechanisms involved during epithelial-mesenchymal transition. Bioessays 23: 912-923, 2001.

10. Boyer B, Valles AM and Edme N: Induction and regulation of epithelial-mesenchymal transitions. Biochem Pharmacol 60: 1091-1099, 2000.

11. Thiery JP: Epithelial-mesenchymal transitions in tumour progression. Nat Rev Cancer 2: 442-454, 2002.

12. Yang AD, Camp ER, Fan F, et al: Vascular endothelial growth factor receptor-1 activation mediates epithelial to mesenchymal transition in human pancreatic carcinoma cells. Cancer Res 66: 46-51, 2006.

13. Elliott BE, Hung WL, Boag AH and Tuck AB: The role of hepatocyte growth factor (scatter factor) in epithelial-mesenchymal transition and breast cancer. Can J Physiol Pharmacol 80: 91-102, 2002.

14. Yang AD, Fan F, Camp ER, et al: Chronic oxaliplatin resistance induces epithelial-to-mesenchymal transition in colorectal cancer cell lines. Clin Cancer Res 12: 4147-4153, 2006.

15. Hiscox S, Jiang WG, Obermeier K, et al: Tamoxifen resistance in MCF7 cells promotes EMT-like behaviour and involves modulation of beta-catenin phosphorylation. Int J Cancer 118: 290-301, 2006.

16. Kajiyama H, Kikkawa F, Khin E, Shibata K, Ino K and Mizutani S: Dipeptidyl peptidase IV overexpression induces up-regulation of E-cadherin and tissue inhibitors of matrix metalloproteinases, resulting in decreased invasive potential in ovarian carcinoma cells. Cancer Res 63: 2278-2283, 2003.

17. Faleiro-Rodrigues C, Macedo-Pinto I, Pereira D and Lopes CS: Prognostic value of E-cadherin immunoexpression in patients with primary ovarian carcinomas. Ann Oncol 15: 1535-1542, 2004.

18. Davidson B, Gotlieb WH, Ben-Baruch G, et al: E-Cadherin complex protein expression and survival in ovarian carcinoma. Gynecol Oncol 79: 362-371, 2000.

19. Darai E, et al: Expression of cadherins in benign, borderline, and malignant ovarian epithelial tumors: a clinicopathologic study of 60 cases. Hum Pathol 28: 922-928, 1997.

20. Agarwal R and Kaye SB: Ovarian cancer: strategies for overcoming resistance to chemotherapy. Nat Rev Cancer 3: 502-516, 2003.

21. Comijn J, Berx G, Vermassen P, et al: The two-handed E box binding zinc finger protein SIP1 downregulates E-cadherin and induces invasion. Mol Cell 7: 1267-1278,2001.

22. Wang X, Ling MT, Guan XY, et al: Identification of a novel function of TWIST, a bHLH protein, in the development of acquired taxol resistance in human cancer cells. Oncogene 23: 474-482, 2004. 